\title{
Ductile Fracture by Development of Surfaces of Unstable Cavity Expansion
}

\author{
C. A. Berg* \\ Institute for Basic Standards, National Bureau of Standards, Washington, D.C. 20234
}

(March 31, 1972)

\begin{abstract}
A model of fracture of a ductile metal in which surfaces of unstable microcavity expansion occur is proposed. It is shown that such surfaces can occur only in locally plane incremental strain. The hardening conditions required for the development of these surfaces are considered. It is shown that in a material possessing an equivalent yield stress $(\mathrm{Y})$ which depends upon the total plastic dilatation $(\Delta)$ and equivalent distortional strain $(\epsilon)$, the minimum ratio of dilatational softening $(\gamma / \Delta<0)$ to distortional hardening $(\gamma / \epsilon>0)$ under which an unstable surface of a dilatation may form is $-2 / 3$. A possible explanation, based upon the model, for the lack of correlation between plane strain ductility and ductility as measured in a tensile test is offered. Also, the pertinence of the model to fracture of glassy polymers and environmental stress cracking of crystalline polymers is discussed.
\end{abstract}

Key words: Ductile fracture; plasticity; void growth.

\section{Introduction}

The chief mechanism of ductile fracture in metals is the expansion, interaction and eventual coalescense of microvoids in regions of large plastic strain and high triaxial stress, (see e.g., Puttick 1959 [1] ${ }^{1}$, Bluhm and Morrissey, 1966 [2]). The cavity growth which produces fracture appears to take place only on the fracture surface itself, with little or no cavity expansion having taken place to either side of the fracture surface (Hayden and Floreen, 1959 [3]). This indicates that the fracture surface is the locus of instability of plastic cavity dilatation, rather than being merely a surface upon which local accidents of e.g., cavity spacing and hardness leads to fracture extension through a field of uniformly dilating cavities. In the following a theoretical model of fracture by the formation of surfaces of unstable cavity growth is considered, and certain elementary but fundamental considerations concerning the orientation of these surfaces and the requirements of hardening to produce such surfaces are discussed.

\footnotetext{
* Professor and Chairman, Department of Mechanical Engineering, The University of Pittsburgh. Presently visiting scientist, Institute for Basic Standards, National Bureau of Standards, Gaithersburg, Maryland.

' Figures in brackets indicate literature references at the end of this paper.
}

It is also pointed out that the mechanism of unstable cavity expansion, which is examined here in connection with fracture of metals, may govern the mechanics of environmental stress cracking of polymers.

\section{Material Behavior}

The material under consideration is a ductile metal or polymer which contains numerous microcavities. Some of these cavities may have been produced during forming processes. Other cavities may be continuously generated in the metal by e.g., cracking of inclusions or separation of phase boundaries in regions of high triaxial stress (near notch tips, or in necks of tensile specimens). The mechanism of fracture under consideration here entails two stages. First, the density and size of microcavities are assumed to increase as deformation of the material proceeds. The cavity growth in this stage is assumed to take place approximately uniformly throughout material elements which are large compared with the mean spacing between cavities but which are still small compared with the size of the specimen under consideration or the scale of length over which gradients of e.g., strain in the specimen are measured. This stage of deformation may 
be called diffuse cavity growth. The second stage of deformation entails the development of an instability in which cavity dilatation localizes in a preferred thin zone, and all deformation ceases to either side of this zone. This stage of deformation, which may be called localization, is tantamount to fracture. The object of the present writing is to clarify the conditions under which transition from diffuse cavity growth to localization may occur.

The actual material in between microcavities is a metal which undergoes large plastic strain by dislocation motion and therefore deforms according to a local yield locus and an associated flow rule (Bishop and Hill, 1951) [4]. An element of microporous material is shown in figure 1. Provided that diffuse cavity growth takes place throughout the element, and provided that the displacement increment $(\delta u)$ and the local tractions $(t)$ acting on the material surfaces of the element are statistically uncorrelated, then (Bishop and Hill, 1951 [4]) the element may be treated as a point in a continuum, subject to stress components $\left(\sigma_{i j}\right)$ equal to appropriate averages of the local tractions acting at the surface of the element, and strain increments $\left(\delta \epsilon_{i j}\right)$ equal to appropriate averages of the incremental displacements $(\delta u)$ at the surface of the element. ${ }^{2}$ In particular, Bishop and Hill [4] demonstrated that because on a local scale the material obeys a yield locus and an associated flow rule, the material element of figure 1 will also obey a yield locus and an associated flow rule.

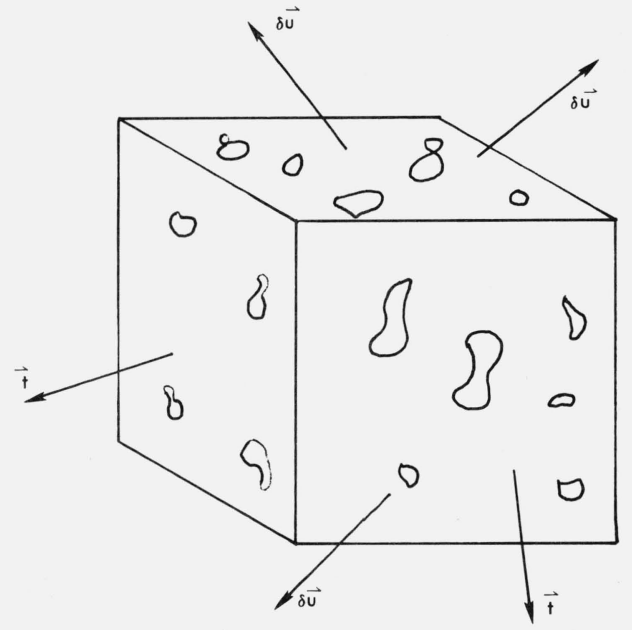

Figure 1: An element of microporous material; local incremental displacements

$(\overrightarrow{\delta u})$ and tractions $(\vec{t})$ at the surface of the element are shown.

The condition for yielding of the microporous element may be given as

$$
f\left(\sigma_{i j}\right)=0,
$$

\footnotetext{
2 The averages employed by Bishop and Hill are given in the appendix.
}

where the $\sigma_{i j}$ are obtained by averaging the local tractions. The plastic strain increments $\delta \epsilon_{i j}{ }^{p}$ obtained by averaging the local surface displacement increments $(\delta u)$ on the element, are given by the associated flow rule

$$
\delta \epsilon_{i j}^{p}=\delta \lambda \frac{\partial_{f}}{\partial \sigma_{i j}},
$$

where $\delta \lambda$ is a scalar multiplier. Since the present writing concerns elements of microporous metal which undergo substantial plastic strain it is assumed that the distinction between the plastic and the elastic strain increment need not be retained. Thus eq (2) may be used to represent the total strain increment.

Since the cavities in the microporous metal element will expand when sufficient triaxial loading is applied to the surface of the element the yield locus of the element will depend upon the mean triaxial stress. The element will exhibit plastic dilational strain which will be represented by the associated flow rule, eq (2).

It is likely that the array of cavities in the element may have some preferred orientation, deriving both from the history of forming of the part and from the history of plastic strain to which the part has been subjected. The primary concern in this writing is the processes of crack nucleation and extension in ductile materials. One must bear in mind that the large plastic strain which occurs in the vicinity of a notch tip will in all likelihood produce strongly oriented arrays of microcavities. The character of anisotropy which should be attributed to the microporous element in order to reflect preferred orientation of the microcavity array is currently under investigation by several workers in the field of fracture. For the present we shall neither assume any special form for the strain history dependent anisotropy or dilatational properties of the microporous ductile element, nor shall we attempt to employ any of the tentative results of present theoretical studies of this problem (e.g., Subudhi, 1970 [5]). For present purposes it will be sufficient to write the yield condition of the element as in eq (1), with the understanding that all six stress components $\left(\sigma_{i j}\right)$ may appear independently in the yield function, and that the plastic dilatation is given by

$$
\delta \boldsymbol{\epsilon}_{k k}=\delta \lambda \frac{\partial_{f}}{\partial \sigma_{k k}},
$$

and that in general $\delta \epsilon_{k k}$ does not vanish. ${ }^{3}$

\section{Kinematics}

Suppose that diffuse cavity growth in a microporous element is to cease, and is to be replaced by localized

${ }^{3}$ Conventional cartesion tensor notation, with the summation convention, is used here. 
cavity growth on a given surface. If deformation is to cease, except in a thin band of rapidly dilating material represented by this surface, then preservation of continuity of the material between the microcavities requires that the strain increments measured in the surface vanish. Figure 2 shows a band of localized dilation occurring according to the process contemplated here. The surface $S$, which represents the dilating band in a continuum theory of fracture, is shown on the figure. The actual boundary between the material in the dilating band and the nondeforming regions to either side is indicated by the dashed lines. Since the material

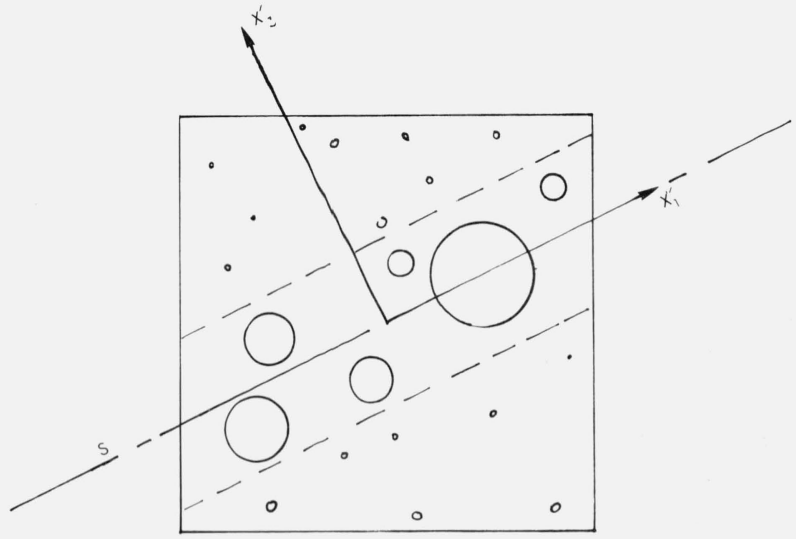

Figure 2: A band of localized cavity expansion along the surface $S$

in the band may not slip with respect to the nondeforming material to either side of the band, the strain components (e.g., $\delta \epsilon_{1.1}, \delta \epsilon_{1.2}, \delta \epsilon_{2.2}$. in figure 2) measured in the surface $S$ must vanish. For the sake of brevity a surface satisfying this requirement may be called a rigid surface. The main point to be recognized here is that the formation of a localized band of cavity dilatation may occur only along a rigid surface.

We now consider the general states of (local) strain increment which permit the rigid surfaces on which localized cavity growth may occur. In general, rigid surfaces may be found only in state of plane incremental strain; while it is obvious that plane deformation may permit rigid surface formation it may be somewhat less than obvious, at least at first inspection, that plane deformation is required for rigid surface formation. To demonstrate this, one may consider the general state of strain increment $\left(\delta \epsilon_{i j}\right)$ which occurs as a rigid surface is formed. Using cartesian coordinates $\left(x_{1}, x_{2}, x_{3}\right)$ with the $x_{1}$ and $x_{2}$ axes lying tangent to the rigid surface, the strain components are

$$
\delta \epsilon_{i j}=\left(\begin{array}{lll}
0 & 0 & \delta \epsilon_{13} \\
0 & 0 & \delta \epsilon_{23} \\
\delta \epsilon_{13} & \delta \epsilon_{23} & \delta \epsilon_{33}
\end{array}\right) .
$$

Thus, the (local) state of incremental strain at the site of a rigid surface consists of longitudinal shear $\left(\delta \epsilon_{13}\right.$, $\left.\delta \epsilon_{23}\right)$ along the direction $\left(x_{3}\right)$ normal to the rigid surface, superposed upon a simple uniaxial strain $\left(\delta \epsilon_{33}\right)$ along the same axis. By a suitable rotation of the $x_{1}, x_{2}$ axes about the $x_{3}$ axis (i.e. $x_{1}{ }^{\prime}=\cos \theta x_{1}+\sin \theta x_{2} ; x_{2}{ }^{\prime \prime}{ }^{\prime \prime} \sigma \iota \nu \theta x_{1}$ $+\cos \theta \cdot \chi_{2} ; x_{3}{ }^{\prime}=x_{3}$, with $\left.-\theta=\tan ^{-1}\left(\delta \epsilon_{13} / \delta \epsilon_{23}\right)\right)$ one obtains a set of coordinates $x_{1}{ }^{\prime}, x_{2}{ }^{\prime}, x_{3}{ }^{\prime}$ with the $x_{3}{ }^{\prime}$ direction still normal to the (local) rigid surface, and in which the components of the strain increments are

$$
\delta \epsilon_{i j}^{\prime}=\left(\begin{array}{lll}
0 & 0 & 0 \\
0 & 0 & \delta \gamma \\
0 & \delta \gamma & \delta \epsilon_{33}
\end{array}\right),
$$

where $(\delta \gamma)^{2}=\left(\delta \epsilon_{13}\right)^{2}+\left(\delta \epsilon_{23}\right)^{2}$. The strain increments state of (5) represent a state of plane incremental deformation in the $x_{2}{ }^{\prime}, x_{3}{ }^{\prime}$ plane; the $\left(x_{1}{ }^{\prime}, x_{2}{ }^{\prime}\right)$ plane, which is the same as the $\left(x_{1}, x_{2}\right)$ plane, lies tangent to the rigid surface.

The conclusion of the above is that in order to nucleate fracture by localization of cavity dilatation at a given point in a specimen, a surface which will permit large scale localized deformation on the surface while deformation to either side of the surface ceases must exist, and this requires that the local mode of deformation be incremental plane strain, with uniaxial strain $(\delta \gamma=0)$ being a special, but, as will be seen, an important case of plane strain.

Additional conditions to complete the kinematic requirements necessary for nucleation of a band of localized dilatation may now be considered. If a band of localized cavity expansion is to form through a field of diffuse cavity expansion in an element under fixed stress, the yield locus of the material in the band will, at the initial instant of localization, be the same as the yield locus of material outside the band. The associated flow rule requires that the strain increments measured at the boundary of the element preserve the same ratios at the instant of localization as obtained at the previous instant, or as would have obtained had localization not occurred. ${ }^{4}$ Since cavity expansion is to occur, the strain increment field immediately prior to localization must contain at least one principal direction along which extension occurs. Recall that a rigid surface can occur only in incremental plane strain. The rigid surface on which localization may nucleate lies perpendicular to the plane of strain, and since the intersection of the rigid surface and the plane of strain must be a line of vanishing extension rate, and the principal strain increments $\left(\delta \epsilon_{1}, \delta \epsilon_{2}\right)$ in the plane of strain represent ex-

${ }^{4}$ In brief, we assume that the yield locus changes continuously, has no sharp corners, and that the strain increment remains normal to the yield locus in an appropriate space. 
trema of extension rates, the two principal strain increments in the plane of strain must be of opposite sign:

$$
\delta \epsilon_{1} \cdot \delta \epsilon_{2} \leqslant 0 \text {. }
$$

The equality in eq (6) would hold for incremental uniaxial strain, in which case one must add the additional requirement that one nonvanishing principal strain increment be greater than zero (e.g., $\delta \epsilon_{1}>0$ ) so that localization of deformation results in cavity expansion rather than compression. For all other cases, eq (9) is sufficient to guarantee the presence of a rigid surface on which localized microcavity expansion is kinematically admissible. It is emphasized that the above pertains to the local kinematic requirements for nucleation of a localized band of cavity expansion, and the requirements stated above are stated in terms of the strain components of the diffuse field of deformation at the inception of the localization instability. The global kinematic requirements for extension of a surface of localization through a field of strain in which all the principal directions of strain are sharply curved, or the strain increment components have strong gradients, have not yet been considered. The requirements given above represent the necessary kinematic conditions for nucleation of fracture by localized cavity growth It is expected that these conditions will be useful in examining both nucleation and progressive extension of fracture, examples of which will be discussed below.

\section{Hardening Requirements}

In order for a band of localized cavity expansion to form in a field of diffuse cavity growth, the stresses in the element must be stationary with respect to the strain increment at the instant of band localization. Otherwise, the material in the incipient band would harden, requiring that further deformation of the band be accompanied by higher stress levels in the band. Since the band formation considered here can occur only in plane strain, the stress in the band and in the surrounding nondeforming material are compatible. ${ }^{5}$ An increase of stress in the band will, at any stage of deformation, require a corresponding increase of stress in the surrounding region. At the inception of banding, when the material in the incipient band has the same yield locus as the surrounding material, hardening of the material in the band would cause deformation of the surrounding material, thus eliminating the band. If the yield locus of the microporous material were represented by

$$
f\left(\sigma_{i j}, I_{1}, I_{2}, \ldots I_{n}\right)
$$

${ }^{5}$ The term compatible is used here in the sense of the limit analysis. The stress in the band is always continuable into the surrounding region because the area of the band does not change. where $I_{1}, I_{2} \ldots I_{n}$ are parameters describing the strain history of the material, ${ }^{6}$ the necessary condition for localization of cavity growth in an element presently being subjected to the kinematically admissible strain increment $\delta \epsilon_{i j}$ would be

$$
\delta f=\frac{\partial_{f}}{\partial I_{r}} \frac{\partial I_{r}}{\partial \epsilon_{i j}} \delta \epsilon_{i j}=0 .
$$

For the practical purposes of estimating, e.g., the mean cavity spacing required in a given material to permit localization under a prescribed strain history and strain increment, eq (8) is of little value unless one has at hand an explicit yield locus and a set of explicit strain history parameters $\left(I_{n}\right)$. Nevertheless, the simple statements of eqs (7) and (8) may prove useful in testing a hypothetical yield locus with respect to internal stability.

It is emphasized that the yield locus need show the stationarity of eq (8) only at the inception of localization. Once localization has developed it would be permissible for the material in the localized band to harden during some later stage of its deformation, provided that the yield locus of the material in the localized band did not increase sufficiently to cause deformation of the material adjacent to the band.

For the purpose of obtaining some concrete estimates of the hardening requirements of localized cavity growth, one may assume that the yield locus does not change shape during deformation and that the hardening of the material may be represented through the net plastic dilatation $\left(\Delta \cong \int \delta \epsilon_{k k}\right)$ and the equivalent distortional strain

$$
\left(\epsilon=\int \sqrt{\frac{2}{3}\left(\delta \epsilon_{i j}-\frac{1}{3} \delta \epsilon_{k k} \delta_{i j}\right)\left(\delta \epsilon_{i j}-\frac{1}{3} \delta_{r r} \delta_{i j}\right)}\right)
$$

which serves as the measure of strain hardening in most practical calculations of conventional plasticity theory. Then the yield co dition may be written as

$$
f\left(\sigma_{i j}\right)-Y(\epsilon, \Delta)=0 .
$$

The hardening requirement for localization eq (8) is then simply

$$
\delta Y=\frac{\partial Y}{\partial \epsilon} \delta \epsilon+\frac{\partial Y}{\partial \Delta} \delta \Delta=0
$$

The assumptions leading to eq (9) certainly do not represent the most general material behavior which one might expect to observe in a microporous element at the point of fracture nucleation. Nevertheless these assumptions do include the basic elements of a theory of dilatational plasticity of microporous material, and

\footnotetext{
${ }^{6}$ This representation would appear to be sufficiently general.
} 
should therefore yield estimates of material behavior which are not entirely unfaithful representations of actual behavior. From eq (10) the condition for localization is

$$
\frac{\partial Y}{\partial \epsilon} / \frac{\partial Y}{\partial \Delta} \cdot=-\frac{\delta \Delta}{\delta \epsilon} .
$$

This expression gives the ratio of the change of yield stress with distortion to the change of yield stress with dilatation, required to permit localization when a given strain increment, having a given ratio of dilatation to distortion is imposed. Dilational strain may either harden or soften the material, depending upon the microcavity density and the rate of hardening of the material between the cavities. Distortional strain, on the other hand, might be expected always to harden the material. ${ }^{7}$ In this case the ratio of distortional hardening to dilational softening required to permit localization of cavity growth in a microporous element subjected to a strain increment having a given ratio of dilation to distortion, is given by eq (11). The strain increment which will permit localization, with the greatest ratio of distortional hardening to dilational softening (i.e., the mode of straining which requires the least softening to permit a localization) is the strain increment which, in addition to meeting the kinematic requirements given previously, provides the maximum ratio of dilatation to distortion; this is just uniaxial extensional strain. Thus, if one wishes to test a given calculational scheme for representing ductile fracture by hole growth in a given array of cavities, it would be useful to consider uniaxial strain, which is the mode of deformation in which fracture by localization of cavity growth may occur earliest. In fact, evaluation of eq (11) for the case of uniaxial incremental strain gives the following estimate of the minimum ratio of dilatational softening $(\partial Y / \partial \Delta<0)$ to distortional hardening hardening $(\partial Y / \partial \epsilon>0)$ which will permit localized cavity dilation.

$$
\left(\frac{\partial Y}{\partial \Delta} / \frac{\partial Y}{\partial \epsilon}\right) \leqslant-\frac{2}{3}
$$

This estimate was also obtained in an earlier writing (Berg, 1969 [6]) in which a special form of the yield locus was assumed.

\section{Orientation of Bands of Localized Cavity Growth}

Since localization of cavity growth can occur only in plane incremental deformation, it is especially con-

7 Exceptions to this generalization may exist in materials with strongly anisotropic microcavity arrays. venient to use the conventional Mohr circle representation of the strain increment in the plane of deformation to show the orientation of surfaces of localization relative to the principal axes of strain increment. Figure 3 shows Mohr circles of (plane) strain increment for several possible cases. Mohr circle I represents a mode of plane strain increment in which the development of localized cavity growth is kinematically possible on either of the planes represented by $a$ or $b$ (since both $a$ and $b$ represent lines of vanishing extension rate in the plane of strain). Circle II represents a state of uniaxial strain increment; in this case the two planes $a$ and $b$ have coalesced at the origin, and thus formation of a single plane of localization is kinematically possible transverse to the axis of incremental strain. The states of incremental strain represented by Circles IV and V contain no directions of vanishing extension rate and therefore do not permit localized cavity growth.

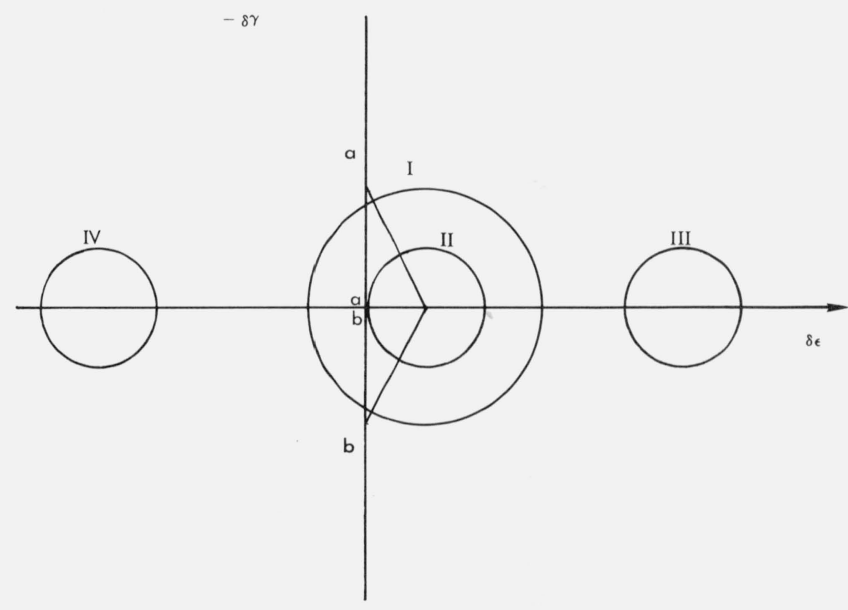

Figure 3: Mohr Circles of incremental strain states; points $a$ and $b$ in circles I and II represent kinematically admissable rigid surfaces.

\section{Discussion}

The model for ductile fracture by localized cavity growth on rigid surfaces may be helpful in explaining some curious phenomena observed in fracture. For example, R. Brook [7] has observed that fracture extending from a notch tip in high strength steels follows a wavy, zig-zag path. Brook's electron micrographs show a fracture surface made up of hillsides and waves, with cavity dimple markings covering the slopes; the average cavity spacing appears to be approximately $1 / 10$ of the length of the slope, or less, so that the present continuum model of fracture by localized cvavity expansion may be applicable. The surfaces on which localization is admissible will, in general, be 
found in conjugate pairs (e.g., the surfaces represented by a and $\mathrm{b}$ in figure 3 ). It appears plausible that the zigzag surfaces observed by Brook were the result of fracture nucleation by localization of cavity growth on one of the two admissible surfaces which occurred in front of the notch, followed or accompanied by nucleation on another surface ahead of the first and lying at the conjugate inclination. This possibility has been discussed earlier (Berg, 1969 [6]).

One of the more striking implications of the model of fracture discussed above is a possible explanation for the general lack of correlation between ductility as measured in uniaxial tension, and ductility as measured in plane strain (Clausing [8]). In the initial stages of deformation of, e.g., a plane strain fracture toughness specimen, the material in the neighborhood of the notch tip will be virtually incompressible, and thus there will be two sets of rigid (kinematically admissible fracture) surfaces at any point. These surfaces will of course be the planes of principal shear strain rate. The deformation at this stage is incompressible, or virtually so; cavity dilation will not be significant, and will not localize. As deformation proceeds microcavity density will increase due both to expansion of cavities initially present and to generation of new cavities through inclusion cracking, phase separation, etc. Throughout this process, as the ratio of dilatation to distortion of each strain increment increases, there will always be two conjugant surfaces, at the notch tip, on which dilatational banding is kinematically admissible. One merely has to wait for that point in the deformation process at which the requirements of hardening (e.g., eq (11)) for localization are met, in order for a band of localized dilatation - and fracture - to occur.

On the other hand, the material in the neck of a tensile specimen undergoes symmetric deformation, with extension occurring along the specimen axis and biaxial contraction occurring transverse to that axis. In this mode of deformation no kinematically admissible surfaces for localized cavity expansion will occur until the material at the center of the neck has suffered sufficient cavity expansion to permit a uniaxial strain increment. If, at this point in the process the requirements of hardening for localization (e.g., eq (12)) have been met, fracture will occur. However, if these requirements have not yet been met, localization will not occur, and the element at the center of the neck may begin to deform with symmetric biaxial extension occurring transverse to the specimen axis (depending upon the rate of cavity growth and nucleation in the material). In this case, the center of the neck will no longer be able to serve as a nucleation site for the lo- calization, and some other point in the neck must serve that purpose.

Thus, with respect to fracture by localization of cavity growth, the plane strain fracture toughness specimen (or any other plane strain specimen) and the uniaxial tension specimen start from different points and arrive at the necessary conditions for fracture by different routes. In plane strain, the kinematic conditions for localization are satisfied from the outset of deformation; one need only wait for the hardening conditions to be met for localization-and fracture-to occur. In the neck of a tensile specimen, the kinematic conditions for nucleation of fracture by localization of cavity growth cannot be satisfied until substantial cavity growth (sufficient to permit uniaxial strain) has occurred at the center of the neck. If the hardening requirements for localized cavity growth are not met at that juncture, the material in the neck may proceed to deform and might possibly begin to undergo biaxial expansion, eliminating the center of the neck as a potential nucleation cite for fracture by localized cavity growth. The apparent lack of correlation between plane strain ductility and uniaxial tensile ductility is therefore not entirely surprising.

\section{Fracture of Polymers}

Cracking in glassy polymers (Orowan and Doyle, 1971 [9]) and stress cracking in certain crystalline polymers is preceded by crazing, which itself is a form of localized cold drawing (plastic deformation) entailing dilatation attributable on the microscopic scale to void formation and expansion. The general conditions given above for fracture of du tile metals by formation of surfaces of unstable void growth should apply as well (with perhaps some suitable modification to the description of the yield locus) to those modes of fracture of polymers which are preceded by crazing. In particular, the mechanical properties, such as yield drop and hardening rate, which determine the sensitivity of given crystalline polymers to environmental stress cracking might be better understood if considered in relation to the model of fracture proposed above.

The author is pleased to acknowledge helpful comments of Professor F. A. McClintock (MIT). In addition the assistance of Mr. H. H. Cheng whose careful reading of an early version of this paper led to simplification of certain of the arguments, is gratefully acknowledged.

\section{References}

[1] Puttick, K. E., Ductile Fracture in Metals, Phil. Mag. 4, pp. 964-969 (1959). 
[2] Bluhm, J. I. and Morrissey, R. J. , Fracture in a Tensile Specimen, Proc. First Internat'l Cong. Fracture Sendai 3, pp. 17-39 (1966).

[3] Hayden, H. W. and Floreen, S., Observations of Localized Deformation During Ductile Fracture, Acta Met 17, pp. 213224 (1969).

[4] Bishop, J. F. W. and Hill, R., A Theory of Plastic Distortion of a Polycrystalline Aggregate Under Combined Stresses, Phil. Mag. 42, pp. 414-427 (1951).

[5] Subudhi, M., M.S. Thesis, Dept. of Mech. Eng. MIT (1970).

[6] erg, C. A., Plastic Dilatation and Void Interaction, Inelastic Behavior of Solids, McGraw Hill, N. Y. (1970).

[7] Brook, R., Proc. Internat'l Cong. on Mech. Prop. Solids, Kyoto, Japan, (in press 1971

[8] Clausing, D. P., Effect of Plastic Strain State on Ductility and Toughness, Internat'l. J. Fracture Mech. 1, pp. $71-85$ (1970).

[9] Orowan, E. and Doyle, M., Striations on Polymer Fracture Surfaces Caused by Rapid Oscillations of the Fracture Mechanism, MIT Industrial Liason Report, MIT, Cambridge, Mass. (1971).

[10] Subudhi, M., Plasticity of Anisotropic Porous Metals, M.S. Thesis, Mech. Engr. Dept., MIT, Cambridge, Mass. (1970).

\section{Appendix}

The method of averaging local (micro) tractions and incremental displacements over the surface of a material element, to provide average stresses $\left(\sigma_{i j}\right)$ and strain increments $\left(\delta \epsilon_{i j}\right)$, which was employed by Bishop and Hill (1951) is as follows.

The local tractions on a small area $\left(S_{j}\right)$ normal to the $x_{j}$ axis are used to obtain the average stress component via

$$
\sigma_{i j}=\frac{1}{S_{j}} \int t_{i} d s_{j}
$$

The local displacement increments $\left(\delta u_{i}\right)$ acting over the entire surface of a unit volume of material bounded by the surface $S$ on which the outward normal vector is denoted by $n_{j}$ are used to obtain the components of average strain increment $\left(\delta \epsilon_{i j}\right)$ via

$$
\delta \epsilon_{i j}=\frac{1}{2} \int_{s}\left(n_{i} \delta u_{j}+n_{j} \delta u_{i}\right) d s
$$

(Paper 76Cl\&2-330) 